\title{
Neoadjuvant Chemotherapy Reduces the Treatment-free Interval After First-line Treatment in Patients With Advanced Ovarian Cancer
}

\author{
ANNE WENG EKMANN-GADE ${ }^{1}$, CLAUS KIM HOGDALL ${ }^{1}$, \\ SVEND AAGE ENGELHOLM ${ }^{2}$ and CARSTEN LINDBERG FAGO-OLSEN ${ }^{1}$ \\ ${ }^{1}$ Department of Gynecology, The Juliane Marie Center, Rigshospitalet, \\ Copenhagen University Hospital, Copenhagen, Denmark; \\ ${ }^{2}$ Department of Oncology, The Finsen Center, Rigshospitalet, \\ Copenhagen University Hospital, Copenhagen, Denmark
}

\begin{abstract}
Background/Aim: The aim of the study was to compare platinum resistance and treatment-free interval (TFI) following treatment with neoadjuvant chemotherapy (NACT) and interval debulking surgery (IDS) or primary debulking surgery $(P D S)$ in women with advanced epithelial ovarian cancer (EOC). Patients and Methods: The study included patients diagnosed with primary EOC, stage IIIC or IV, between 2005 and 2013. Patients were grouped according to first-line treatment (PDS vs. NACT-IDS). Date of second-line treatment initiation was used to evaluate platinum sensitivity. Results: The study population included 521 patients, of which 371 (71\%) and 150 (29\%) underwent PDS and NACT-IDS, respectively. We found no difference in platinum resistance between groups. Platinum-sensitive patients treated with NACT-IDS had a shorter median TFI (372 vs. 497 days, $p=0.042$ ). Similarly, patients with no residual tumor after IDS had a shorter median TFI (280 vs. 302 days, $p=0.005)$. Conclusion: NACT-IDS may shorten the TFI after first-line platinum-based chemotherapy.
\end{abstract}

Epithelial ovarian cancer (EOC) is the leading cause of death among gynecological malignancies $(1,2)$. Most women are diagnosed in advanced stages (The International Federation of Gynecology and Obstetrics (FIGO) stage III-IV), for which the

This article is freely accessible online.

Correspondence to: Anne Weng Ekmann-Gade, Department of Gynecology, The Juliane Marie Center, Rigshospitalet, Copenhagen University Hospital, Blegdamsvej 9, 2100 Copenhagen, Denmark. Tel: +45 30346768, e-mail: anne.weng.ekmann-gade@regionh.dk

Key Words: Ovarian cancer, neoadjuvant chemotherapy, treatmentfree interval, platinum resistance. prognosis is poor (3). The traditional treatment for EOC is primary debulking surgery (PDS) followed by adjuvant platinum-based chemotherapy (4). However, in the last decade, the use of neoadjuvant chemotherapy (NACT) followed by interval debulking surgery (IDS) has increased $(5,6)$.

For PDS, complete tumor resection has a profoundly positive impact on survival $(7,8)$. However, while randomized controlled trials comparing PDS to NACT-IDS demonstrated superior surgical outcomes for NACT-IDS, this finding did not translate into better survival $(9,10)$. Another important factor regarding survival is platinum sensitivity to first-line chemotherapy since platinumresistant disease is associated with poor prognosis $(4,11$ 13). The potential risk of developing platinum resistance rises with increasing tumor burden (14). Accordingly, patients treated with chemotherapy before debulking surgery may be more susceptible to developing resistance to chemotherapy.

Hypothetically, the reason why the superior surgical outcomes of NACT-IDS do not translate into better survival could in part be explained by a greater likelihood of developing platinum resistance when treated with NACTIDS than when treated with PDS. Thus, the present study compared platinum resistance and treatment-free interval (TFI) after first-line treatment with PDS or NACT-IDS.

\section{Patients and Methods}

Study population. This study included patients treated at Copenhagen University Hospitals, Rigshospitalet or Herlev Hospital, and identified in the Danish Gynecological Cancer Database (DGCD) (15) between January 2005 and May 2013 with primary epithelial stage IIIC or IV cancer in the ovaries, fallopian tubes, or peritoneum. We selected two university hospitals from the greater Copenhagen region, as they have the same medical record system, allowing us to validate data through review of medical records. 
Table I. Baseline characteristics of the study population.

\begin{tabular}{|c|c|c|c|c|}
\hline & $\begin{array}{c}\text { PDS } \\
\mathrm{n}=371 \\
(71 \%)\end{array}$ & $\begin{array}{c}\text { NACT-IDS } \\
\mathrm{n}=150 \\
(29 \%)\end{array}$ & $\begin{array}{c}\text { Total } \\
\mathrm{n}=521\end{array}$ & $p$-Value \\
\hline Age, median (IQR) & $64(55-72)$ & $65(57-72)$ & $64(56-72)$ & 0.23 \\
\hline Stage IV disease, $\%(\mathrm{n})$ & $19(69)$ & $53(80)$ & $29(149)$ & 0.00 \\
\hline BMI, median (IQR) & $24 * a(22-27)$ & $24 * b(22-29)$ & $24 * \mathrm{c}(22-27)$ & 0.44 \\
\hline No co-morbidity, $\%$ (n) & $64 * \mathrm{~d}(234)$ & $61 * \mathrm{e}(89)$ & $63 * f(323)$ & 0.54 \\
\hline \multicolumn{5}{|l|}{ ASA score $>2$} \\
\hline Proportion of $>2, \%(n)$ & $14^{*} \mathrm{~g}(52)$ & $16 * \mathrm{~h}(23)$ & $15 * \mathrm{i}(75)$ & 0.49 \\
\hline \multicolumn{5}{|l|}{ ECOG performance status } \\
\hline Proportion of $>2, \%(n)$ & $1 * \mathrm{j}(3)$ & $2 * \mathrm{k}(3)$ & $1 * 1(6)$ & 0.35 \\
\hline \multicolumn{5}{|l|}{ Histology } \\
\hline Serous histology, \% (n) & $81(302)$ & $92(138)$ & $85(440)$ & 0.02 \\
\hline No residual tumor after surgery, \% (n) & $39(144)$ & $58(87)$ & $44(231)$ & 0.00 \\
\hline
\end{tabular}

PDS: Primary debulking surgery; NACT: neoadjuvant chemotherapy; IDS: interval debulking surgery; IQR: interquartile range; ASA: American Society of Anesthesiologists; ECOG: The Eastern Cooperative Oncology Group. *a-g: Missing data on: *a=5, *b=4, *c=9, *d=7, *e=4, *f=10, * $\mathrm{g}=1$, $* \mathrm{~h}=10, * \mathrm{i}=11, * \mathrm{j}=1, * \mathrm{k}=10, * \mathrm{l}=11$.

Definitions of treatment groups and treatments. The patients were categorized into two groups according to their primary treatment. The first group included patients treated with PDS, as an initial surgical attempt to remove the tumor, before receiving at least one cycle of platinum-based chemotherapy. The second group included patients treated with NACT-IDS who received one to four cycles of platinum-based chemotherapy before IDS and at least one cycle of platinum-based chemotherapy after the surgery according to national and international treatment regimens $(4,16,17)$. Chemotherapy response was defined as sensitive if the patient started second-line treatment more than 6 months after the last firstline treatment. Chemotherapy response was defined as resistant if the patient started second-line treatment during or within 6 months after the last first-line treatment. TFI was defined as the time from the last first-line chemotherapy treatment to the first second-line chemotherapy treatment. For patients without second-line treatment, the last day of follow-up was June 1, 2015. All chemotherapy treatment dates were obtained from the DGCD and validated by medical record review.

According to national guidelines from the Danish Gynecological Cancer Group, patients are referred to NACT-IDS if preoperative evaluation suggests that complete tumor debulking is unachievable; that is, for patients with a spread of disease that is estimated to be unresectable or patients with severe co-morbidity and/or age above $75-80$ years (16). Patients who had two debulking surgeries during their first-line chemotherapy were excluded.

The chemotherapeutic treatment followed Danish national guidelines, in line with international regimens and consisted of intravenous carboplatin and taxane or carboplatin monotherapy. Apart from the timing, there was no difference between adjuvant and neoadjuvant chemotherapy regimens. Clinical oncologists, subspecialized in gynecological malignancies, prescribed the chemotherapy treatments.

For both PDS and IDS, specialists in gynecological cancer surgery performed the operations. The standard debulking procedures included total hysterectomy, bilateral salpingooophorectomy, omentectomy, appendectomy, and lymphadenectomy.
Table II. Platinum resistance and sensitivity for the entire study population and in patients with no residual tumor after surgery.

\begin{tabular}{|c|c|c|c|c|}
\hline & \multicolumn{3}{|c|}{$\begin{array}{l}\text { Entire study population } \\
\qquad(\mathrm{n}=521)\end{array}$} & \multirow[b]{2}{*}{$p$-Value } \\
\hline & PDS & NACT-IDS & Total & \\
\hline Platinum resistance, $\%$ (n) & $33(122)$ & $35(53)$ & 175 & \\
\hline Platinum sensitivity, \% (n) & 67 (249) & $65(97)$ & 346 & 0.61 \\
\hline \multirow[t]{3}{*}{ Total } & 371 & 150 & 521 & \\
\hline & \multicolumn{3}{|c|}{$\begin{array}{l}\text { Patients with no residual } \\
\text { tumor after surgery }(n=231)\end{array}$} & \\
\hline & PDS & NACT-IDS & Total & $p$-Value \\
\hline Platinum resistance, $\%$ (n) & $19(27)$ & $18(16)$ & 43 & \\
\hline Platinum sensitivity, \% (n) & 81 (117) & $82(71)$ & 188 & 0.95 \\
\hline Total & 144 & 87 & 231 & \\
\hline
\end{tabular}

PDS: Primary debulking surgery; NACT: neoadjuvant chemotherapy; IDS: interval debulking surgery; Platinum resistance=treatment free interval $<6$ months; Platinum sensitivity=treatment free interval $>6$ months.

Explorative laparotomy with biopsy only or palliative omentectomy was not considered debulking surgery.

The operating gynecologist estimated the presence of residual tumor after surgery and gynecological pathologists evaluated the surgical specimens. Performance status was evaluated using the Eastern Cooperative Oncology Group (ECOG) criteria. Comorbidity was defined as any concurrent disease at the time of registration in the DGCD.

Data sources. This population-based cohort study analyzed data from the DGCD, a nationwide multidisciplinary database that 
Table III. Multivariate logistic regression analyses for platinum resistance.

\begin{tabular}{lccc}
\hline & OR & $95 \%$ CI & $p$-Value \\
\hline NACT-IDS $v s$. PDS & 1.48 & $0.93-2.37$ & 0.10 \\
Stage IV $v s$. IIIC & 1.44 & $0.92-2.24$ & 0.11 \\
Other histology $v s$. serous histology & 2.63 & $1.55-4.47$ & 0.00 \\
Residual tumor $v s$. no residual tumor after surgery & 4.32 & $2.81-6.64$ & 0.00 \\
\hline
\end{tabular}

OR: Odds ratio; CI: confidence interval; PDS: primary debulking surgery; NACT: neoadjuvant chemotherapy; IDS: interval debulking surgery; platinum resistance=treatment-free interval < six months; vs.: versus.

contains detailed information about all types of gynecological cancer. All gynecological, pathological, and oncological departments in Denmark that take part in the diagnosis and treatment of gynecological malignancies are obligated to report to the DGCD. The medical records were examined for all patients for validation of chemotherapy-specific data.

Statistical analysis. Age and body mass index (BMI) were considered continuous variables and comparisons between groups were performed using Mann-Whitney $U$-tests. Treatment groups (PDS or NACT-IDS), stage of disease (IIIC or IV), presence of comorbidity (yes or no), American Society of Anesthesiologists (ASA) score $(\leq 2$ or $>2$ ), ECOG performance status $(\leq 2$ or $>2)$, histology (serous or others), presence of residual tumor after surgery (yes or no), and platinum sensitivity $v s$. platinum resistance were considered categorical data and comparisons between groups were performed using chi-square tests. Multivariate logistic regression was conducted using platinum resistance as a dependent variable. Significant differences in the univariate statistical tests were included as independent variables. TFIs were compared between groups by the Kaplan-Meier method and log-rank analyses. Since we suspected that patients treated with NACT were only referred to IDS if they were good responders, differences in treatment groups were likely to exist. Thus, the TFIs were compared for both patients with platinum-sensitive disease and those with no residual tumor after surgery.

Statistical analyses were performed using Statistical Package for the Social Sciences (SPSS) for Windows, version 22.0. Two-sided $p$-values of $<0.05$ were considered statistically significant.

\section{Results}

The study population included 521 patients with stages IIIC and IV EOC. Among these, 371 (71\%) patients underwent PDS and 150 (29\%) underwent NACT-IDS. Table I presents the baseline characteristics of the study population. Patients treated with NACT-IDS, more often presented with stage IV disease, serous histology, and no residual tumor after surgery.

At 6 months from the completion of the initial platinumbased chemotherapy, $121(33 \%)$ of the patients in the PDS group had started second-line chemotherapy and were considered to have platinum-resistant disease in comparison to $53(35 \%)$ patients in the NACT-IDS group $(p=0.61)$ (Table II). Two hundred-and-thirty-one patients (44\%) had no residual tumor after surgery. Of these, 27 patients (19\%) in the PDS group had platinum-resistant disease, which did not differ significantly from the 16 patients $(18 \%)$ in the NACT-IDS group with platinum-resistant disease $(p=0.95)$ (Table II).

A multivariate logistic regression analysis (Table III) confirmed that the timing of primary chemotherapy was not associated with an increased risk of platinum resistance. However, histology other than serous histology (odds ratio $2.63,95 \%$ confidence interval $=1.55-4.47$ ) and residual tumor after surgery (odds ratio $4.32,95 \%$ confidence interval $=2.81$ 6.64) were risk factors for the recurrence of platinumresistant disease.

Patients with platinum-sensitive disease treated with NACT-IDS had a significantly shorter median TFI than patients treated with PDS (372 vs. 497 days; $p=0.042)$. The median TFI was significantly shorter for patients with no residual tumor after IDS than that for patients with no residual tumor after PDS, (280vs. 302 days, $p=0.005)$.

\section{Discussion}

Although studies have shown a better surgical outcome of IDS than that for PDS regarding residual tumor, this does not seem to translate into better survival in patients treated with NACT-IDS; thus, it remains controversial whether NACTIDS compromises survival compared to PDS. However, the results might be confounded by the administration of NACTIDS to very frail patients or patients with more advanced disease, which leads to shorter survival.

In the present study, NACT-IDS was not associated with an increased risk of platinum resistance in both univariate and multivariate regression analyses. Few other, considerably smaller, retrospective trials have studied platinum resistance following PDS and NACT-IDS (18-21). Similar to our results, da Costa et al. found no difference in platinumresistant disease (19). In contrast, Rauh-Hain et al. reported a higher rate of platinum-resistant disease among patients treated with NACT-IDS. However, after adjusting for other prognostic factors, the difference was not statistically significant (18). Correspondingly, Petrillo et al. (20) observed a higher rate of platinum-resistant disease after NACT-IDS 
than after PDS, but only in univariate analysis. Lastly, Luo et al. (21) found that patients treated with NACT-IDS had a higher incidence of platinum-resistant disease than patients treated with PDS, which was also confirmed in a multivariate regression analysis. However, the NACT-IDS group in the study by Luo et al. was relatively small.

Few studies have investigated platinum resistance at the second recurrence $(18,19)$. Although no significant difference in platinum-resistant disease was observed at the first recurrence in the studies by Rauh-Hain and da Costa, the risk of platinum resistance at the second recurrence was significantly higher in patients treated with NACT-IDS in both studies. Therefore, patients initially treated with NACTIDS might have an increased risk of platinum-resistant disease later in their disease course. Thus, the timeline could explain why the present study did not observe a significant difference in platinum resistance between the two treatments.

The statistical likelihood of a secondary response to primary platinum-based chemotherapy increases with increasing TFI (22). Our results showed a significantly shorter median TFI in patients with platinum-sensitive disease and those with no residual tumor after surgery who were treated with NACT-IDS than that in patients who received PDS. Therefore, even with the best possible surgical outcome of IDS, the effect of NACT may still be impaired compared to that of PDS. This may be explained by the fact that the larger the volume of cancer present when chemotherapy is initiated, the higher the likelihood of the development of mutations and resistance to chemotherapy (14). Consequently, patients receiving NACT might show a poorer response to platinum-based chemotherapy due to the exposure of a larger tumor burden to chemotherapy before IDS. In this context, NACT may induce chemotherapy resistance in colonies of cancer stem cells that are difficult to detect after chemotherapy and, therefore, difficult to remove at IDS (23). Hynninen et al. (24) demonstrated that visual examination of the abdominal cavity is more difficult in patients treated with NACT than in patients never exposed to chemotherapy. Thus, NACT may increase the risk of leaving small tumor colonies and the rate of complete debulking may be overestimated. This hypothesis is supported by the results of a recent study by Himoto et al., which revealed that patients treated with NACT-IDS were more likely to experience recurrence in the same sites as the original disease, whereas patients who underwent PDS had higher number of recurrences in new locations; away from the surgically cleared sites (25). In line with this, two other studies have shown that large tumor burdens impair chemotherapy response $(26,27)$.

The number of patients with no comorbidity is slightly lower in the NACT-IDS group compared to the PDS group (Table I). Since the presence of significant co-morbidity is an indication for referral to NACT, we expected the opposite.
This may be due to the study design since the patients with co-morbidities and in poor medical conditions, treated with NACT are not referred to IDS, expectantly. The results from the group of NACT-IDS patients in the present study are thus likely to be biased toward greater probability of platinum sensitivity and longer TFIs.

The differences in the proportion of serous histology between PDS and NACT-IDS groups are $81 \%$ and $92 \%$, respectively. However, patients are not selected for either PDS or NACT-IDS based on histology but rather based on national guidelines. Thus, the difference in histology between the two treatment groups did not influence the choice of first line treatment. Yet, the response to treatment may have been affected by the histology.

TFI is the most widely used and accepted clinical surrogate for predicting chemotherapy response and prognosis (28). In case of recurrence, the response to platinum-based chemotherapy is directly associated with the TFI duration. Thus, our finding is important as it indicates that NACT compromises good responders to first-line chemotherapy. This may be an important factor for clinicians to consider when choosing the treatment pathway for their patients.

To our knowledge, the present study is the largest in size on this topic. The data were prospectively registered in the DGCD and validated by a medical record review. Moreover, the patients were categorized to match international treatment regimens. However, some limitations to the study design should be mentioned. The main limitation of this study was its nonrandomized design. Selection bias for IDS and PDS existed. Differences may have occurred during treatment, as patients treated with NACT may only have been referred to IDS if they responded to chemotherapy. Also, we excluded patients who did not undergo surgery. Consequently, the NACT-IDS group may have consisted of a selected group of patients, which could cause bias resulting in longer TFIs and a higher likelihood of platinum sensitivity. To minimize this inherited selection bias that mirrors current clinical practice, we focused on patients with no residual tumor after surgery and patients with platinumsensitive disease only. However, this issue can only be addressed by a prospective randomized study design. Another limitation was our use of the date of the first treatment of the second-line chemotherapy as a surrogate marker for recurrence. Thus, TFI was calculated instead of progression-free interval.

In conclusion, the results of this population-based cohort study showed that NACT-IDS treatment may impair TFI in EOC patients with no residual tumor after surgery and in patients with platinum-sensitive disease. No difference in platinum resistance was observed between patients treated with PDS or NACT-IDS. Still, selection bias for IDS and PDS exists; accordingly, the results of the present study 
should not be considered definitive conclusions but rather hypothesis-generating findings. A prospective study is needed to better derive the effect of NACT-IDS on TFI and the development of platinum resistance.

\section{Conflicts of Interest}

The Authors declare no conflicts of interest regarding this study.

\section{Authors' Contributions}

All Authors made a significant contribution to this study. CLF and AWE drafted the original study protocol. CLF, AWE, CKH and SAE contributed to the analysis and interpretation of the data. AWE drafted the paper, and all Authors commented on and approved the final version and this submission.

\section{Acknowledgements}

The Danish Gynecological Cancer Database is acknowledged for acquisition of all data regarding patients and treatment.

\section{References}

1 Siegel RL, Miller KD and Jemal A: Cancer statistics, 2019. CA Cancer J Clin 69: 7-34, 2019. PMID: 30620402. DOI: $10.3322 /$ caac. 21551 .

2 Sant M, Chirlaque Lopez MD, Agresti R, Sanchez Perez MJ, Holleczek B, Bielska-Lasota M, Dimitrova N, Innos K, Katalinic A, Langseth H, Larranaga N, Rossi S, Siesling S and Minicozzi P: Survival of women with cancers of breast and genital organs in Europe 1999-2007: Results of the EUROCARE-5 study. Eur J Cancer 51: 2191-2205, 2015. PMID: 26421822. DOI: 10.1016/j.ejca.2015.07.022.

3 Annual Report 2016-2017. Danish Gynecological Cancer Database. Nationwide clinical database for cancer in the ovaries, uterus and cervix. Available at: http://www.dgcg.dk/images/Grupper/ Databasegruppen/rsrapport_DGCD_2016-17_endelig_ anonymiseret.pdf

4 Ledermann JA, Raja FA, Fotopoulou C, Gonzalez-Martin A, Colombo $\mathrm{N}$ and Sessa C: Newly diagnosed and relapsed epithelial ovarian carcinoma: ESMO Clinical Practice Guidelines for diagnosis, treatment and follow-up. Ann Oncol 24(Suppl 6): vi24-32, 2013. PMID: 24078660. DOI: 10.1093/annonc/mdt333

5 Fago-Olsen CL, Ottesen B, Kehlet H, Markauskas A, Mosgaard BJ, Ottosen C, Sogaard CH, Sogaard-Andersen E and Hogdall C: Neoadjuvant chemotherapy as ovarian cancer treatment: ever more used with major regional differences. Dan Med J 59: A4477, 2012. PMID: 22849978.

6 Horner W, Peng K, Pleasant V, Brackmann M, Ebott J, Gutfreund R, McLean K, Reynolds RK and Uppal S: Trends in surgical complexity and treatment modalities utilized in the management of ovarian cancer in an era of neoadjuvant chemotherapy. Gynecol Oncol 154: 283-289, 2019. PMID: 31196575. DOI: 10.1016/j.ygyno.2019.05.023

7 Bristow RE, Tomacruz RS, Armstrong DK, Trimble EL and Montz FJ: Survival effect of maximal cytoreductive surgery for advanced ovarian carcinoma during the platinum era: a meta- analysis. J Clin Oncol 20: 1248-1259, 2002. PMID: 11870167. DOI: $10.1200 / J C O .2002 .20 .5 .1248$

8 du Bois A, Reuss A, Pujade-Lauraine E, Harter P, Ray-Coquard I and Pfisterer J: Role of surgical outcome as prognostic factor in advanced epithelial ovarian cancer: a combined exploratory analysis of 3 prospectively randomized phase 3 multicenter trials: by the Arbeitsgemeinschaft Gynaekologische Onkologie Studiengruppe Ovarialkarzin. Cancer 115: 1234-1244, 2009. PMID: 19189349. DOI: $10.1002 /$ cncr.24149

9 Vergote I, Trope CG, Amant F, Kristensen GB, Ehlen T, Johnson N, Verheijen RHM, van der Burg MEL, Lacave AJ, Panici PB, Kenter GG, Casado A, Mendiola C, Coens C, Verleye L, Stuart GCE, Pecorelli S and Reed NS: Neoadjuvant chemotherapy or primary surgery in stage IIIC or IV ovarian cancer. N Engl J Med 363: 943-953, 2010. PMID: 20818904. DOI: 10.1056/NEJMoa0 908806

10 Kehoe S, Hook J, Nankivell M, Jayson GC, Kitchener H, Lopes T, Luesley D, Perren T, Bannoo S, Mascarenhas M, Dobbs S, Essapen S, Twigg J, Herod J, McCluggage G, Parmar M and Swart AM: Primary chemotherapy versus primary surgery for newly diagnosed advanced ovarian cancer (CHORUS): an openlabel, randomised, controlled, non-inferiority trial. Lancet (London, England) 386: 249-257, 2015. PMID: 26002111. DOI: 10.1016/S0140-6736(14)62223-6

11 Markman M and Bookman MA: Second-line treatment of ovarian cancer. Oncologist 5: 26-35, 2000. PMID: 10706647.

12 Herzog TJ and Pothuri B: Ovarian cancer: a focus on management of recurrent disease. Nat Clin Pract Oncol 3: 604611, 2006. PMID: 17080178. DOI: 10.1038/ncponc0637

13 Colombo P-E, Fabbro M, Theillet C, Bibeau F, Rouanet P and Ray-Coquard I: Sensitivity and resistance to treatment in the primary management of epithelial ovarian cancer. Crit Rev Oncol Hematol 89: 207-216, 2014. PMID: 24071502. DOI: 10.1016/j.critrevonc. 2013.08 .017

14 Goldie JH and Coldman AJ: A mathematic model for relating the drug sensitivity of tumors to their spontaneous mutation rate. Cancer Treat Rep 63: 1727-1733, 1979. PMID: 526911.

15 Sorensen SM, Bjorn SF, Jochumsen KM, Jensen PT, Thranov IR, Hare-Bruun $\mathrm{H}$, Seibaek $\mathrm{L}$ and Hogdall C: Danish gynecological cancer database. Clin Epidemiol 8: 485-490, 2016. PMID: 27822089. DOI: 10.2147/CLEP.S99479

16 Danish Gynecological Cancer Group. Instructions for referral, diagnostics and control of epithelial ovarian-, fallopian tubal and primary peritoneal cancer and borderline tumors. Dansk Gynækologisk Cancer Gruppe. Retningslinjer for visitation, diagnostik.

17 Wright AA, Bohlke K, Armstrong DK, Bookman MA, Cliby WA, Coleman RL, Dizon DS, Kash JJ, Meyer LA, Moore KN, Olawaiye AB, Oldham J, Salani R, Sparacio D, Tew WP, Vergote I and Edelson MI: Neoadjuvant chemotherapy for newly diagnosed, advanced ovarian cancer: Society of Gynecologic Oncology and American Society of Clinical Oncology Clinical Practice Guideline. Gynecol Oncol 143: 3-15, 2016. PMID: 27650684. DOI: $10.1016 /$ j.ygyno.2016.05.022

18 Rauh-Hain JA, Nitschmann CC, Worley MJJ, Bradford LS, Berkowitz RS, Schorge JO, Campos SM, del Carmen MG and Horowitz NS: Platinum resistance after neoadjuvant chemotherapy compared to primary surgery in patients with advanced epithelial ovarian carcinoma. Gynecol Oncol 129: 6368, 2013. PMID: 23337490. DOI: 10.1016/j.ygyno.2013.01.009 
19 da Costa AABA, Valadares C V, Baiocchi G, Mantoan H, Saito A, Sanches S, Guimaraes AP and Achatz MIW: Neoadjuvant chemotherapy followed by interval debulking surgery and the risk of platinum resistance in epithelial ovarian cancer. Ann Surg Oncol 22: S971-8, 2015. PMID: 26014155. DOI: 10.1245/s10434-0154623-Z

20 Petrillo M, Ferrandina G, Fagotti A, Vizzielli G, Margariti PA, Pedone AL, Nero C, Fanfani F and Scambia G: Timing and pattern of recurrence in ovarian cancer patients with high tumor dissemination treated with primary debulking surgery versus neoadjuvant chemotherapy. Ann Surg Oncol 20: 3955-3960, 2013. PMID: 23838915. DOI: 10.1245/s10434-013-3091-6

21 Luo Y, Lee M, Kim HS, Chung HH and Song YS: Effect of neoadjuvant chemotherapy on platinum resistance in stage IIIC and IV epithelial ovarian cancer. Medicine (Baltimore) 95: e4797, 2016. PMID: 27603388. DOI: 10.1097/MD.0000000000004797

22 Markman M: Pharmaceutical management of ovarian cancer : current status. Drugs 68: 771-789, 2008. PMID: 18416585. DOI: 10.2165/00003495-200868060-00004

23 Lim MC, Song YJ, Seo SS, Yoo CW, Kang S and Park SY: Residual cancer stem cells after interval cytoreductive surgery following neoadjuvant chemotherapy could result in poor treatment outcomes for ovarian cancer. Onkologie 33: 324-330, 2010. PMID: 20523098. DOI: 10.1159/000313823

24 Hynninen J, Lavonius M, Oksa S, Grenman S, Carpen O and Auranen A: Is perioperative visual estimation of intra-abdominal tumor spread reliable in ovarian cancer surgery after neoadjuvant chemotherapy? Gynecol Oncol 128: 229-232, 2013. PMID: 23142076. DOI: 10.1016/j.ygyno.2012.11.007
25 Himoto Y, Cybulska P, Shitano F, Sala E, Zheng J, Capanu M, Nougaret S, Nikolovski I, Vargas HA, Wang W, Mueller JJ, Chi DS and Lakhman Y: Does the method of primary treatment affect the pattern of first recurrence in high-grade serous ovarian cancer? Gynecol Oncol, 2019. PMID: 31521322. DOI: 10.1016/j.ygyno.2019.08.011

26 Eisenhauer EL, Abu-Rustum NR, Sonoda Y, Aghajanian C, Barakat RR and Chi DS: The effect of maximal surgical cytoreduction on sensitivity to platinum-taxane chemotherapy and subsequent survival in patients with advanced ovarian cancer. Gynecol Oncol 108: 276-281, 2008. PMID: 18063020. DOI: $10.1016 /$ j.ygyno.2007.10.022

27 Yildirim Y, Ertas IE, Dogan A, Gultekin OE and Gultekin E: The predictors of response to neoadjuvant chemotherapy in advanced epithelial ovarian cancer. J Surg Oncol 105: 200-205, 2012. PMID: 21815151. DOI: 10.1002/jso.22053

28 Wilson MK, Pujade-Lauraine E, Aoki D, Mirza MR, Lorusso D, Oza AM, du Bois A, Vergote I, Reuss A, Bacon M, Friedlander M, Gallardo-Rincon D, Joly F, Chang SJ, Ferrero AM, Edmondson RJ, Wimberger P, Maenpaa J, Gaffney D, Zang R, Okamoto A, Stuart G and Ochiai K: Fifth Ovarian Cancer Consensus Conference of the Gynecologic Cancer InterGroup: recurrent disease. Ann Oncol 28: 727-732, 2017. PMID: 27993805. DOI: $10.1093 /$ annonc/mdw663

Received March 13, 2020

Revised March 23, 2020

Accepted March 24, 2020 